\title{
Uniqueness of Symplectic Structures
}

\author{
Dietmar Salamon* \\ ETH Zürich
}

12 December 2012

\begin{abstract}
This survey paper discusses some uniqueness questions for symplectic forms on compact manifolds without boundary.
\end{abstract}

\section{Introduction}

Let $M$ be an oriented $2 n$-manifold. A symplectic form on $M$ is a closed 2form $\omega \in \Omega^{2}(M)$ whose top exterior power $\omega^{n}$ is a volume form. Fundamental questions in symplectic topology are which deRham cohomology classes $a \in H^{2}(M ; \mathbb{R})$ are represented by symplectic forms (the existence problem) and whether any two symplectic forms on $M$ that represent the same cohomology class are related by a suitable equivalence relation (the uniqueness problem). Necessary conditions for the existence of symplectic forms are the existence of an almost complex structure and, in the closed case, the existence of a cohomology class $a \in H^{2}(M ; \mathbb{R})$ with $a^{n}>0$. Whether or not these conditions are also sufficient, when $M$ is a closed manifold, is completely open in dimensions $2 n \geq 6$. In dimension four additional necessary conditions for existence arise from Seiberg-Witten theory. In the present survey paper the focus is mainly on the uniqueness problem. Section 2 gives precise formulations of some relevant questions, Section 3 discusses what is known about these question in some examples (without any claim of providing a complete picture), and Section 4 discusses some conclusions and conjectures as well as the Donaldson geometric flow approach to the uniqueness problem for hyperkähler surfaces.

*Partially supported by the Swiss National Science Foundation Grant 200021-127136 


\section{The space of symplectic forms}

2.1 (Equivalence relations). Let $M$ be a manifold without boundary. Consider the following statements for two symplectic forms $\omega_{0}, \omega_{1}$ on $M$.

(a) $\omega_{0}$ and $\omega_{1}$ are connected by a path of cohomologous symplectic forms.

(b) $\omega_{0}$ and $\omega_{1}$ are connected by a path of symplectic forms.

(c) $\omega_{0}$ and $\omega_{1}$ are connected by a path of nondegenerate 2 -forms.

(d) $\omega_{0}$ and $\omega_{1}$ have the same first Chern class in $H^{2}(M ; \mathbb{Z})$.

(A) There exists a diffeomorphism $\varphi$ of $M$ such that $\omega_{0}=\varphi^{*} \omega_{1}$.

(B) There exists a diffeomorphism $\varphi$ of $M$ such that $\omega_{0}$ and $\varphi^{*} \omega_{1}$ are connected by a path of symplectic forms.

(C) There exists a diffeomorphism $\varphi$ of $M$ such that $\omega_{0}$ and $\varphi^{*} \omega_{1}$ are connected by a path of nondegenerate 2 -forms.

(D) There exists a diffeomorphism $\varphi$ of $M$ such that $c_{1}\left(\omega_{0}\right)=\varphi^{*} c_{1}\left(\omega_{1}\right)$.

These statements define equivalence relations. Two symplectic forms $\omega_{0}$ and $\omega_{1}$ are called isotopic if they are related by (a) (i.e. a path of cohomologous symplectic forms), they are called homotopic if they are related by (b) (i.e. a path of symplectic forms), they are called diffeomorphic if they are related by (A) (i.e. a diffeomorphism), and they are called deformation equivalent if they are related by (B) (i.e. a diffeomorphism, followed by a path of symplectic forms).

For closed manifolds these equivalence relations are related as follows

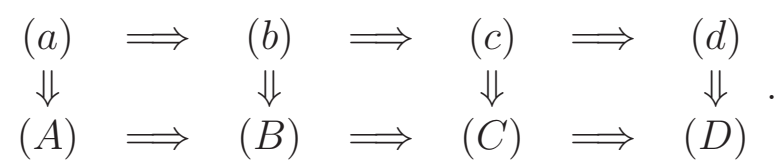

In particular, by Moser isotopy, assertion (a) holds if and only if there exists a diffeomorphism $\varphi: M \rightarrow M$ isotopic to the identity such that $\varphi^{*} \omega_{1}=\omega_{0}$. Thus (a) implies (A). For (A), (B), (C) it may also be interesting to restrict to diffeomorphisms that act as the identity on homology (see Karshon-KesslerPinsonnault [21]). For (A) this would mean that equivalent symplectic forms represent the same cohomology class and for (B) that they have the same first Chern class and the same Gromov-Witten invariants. 
2.2 (Three questions). The uniqueness problem in symplectic topology is the problem of understanding these equivalence relations on the space of symplectic forms. Here are some questions that may serve as a guideline. Fix a deRham homology class

$$
a \in H^{2}(M ; \mathbb{R})
$$

and denote the space of symplectic forms on $M$ representing the class $a$ by

$$
\mathcal{S}_{a}:=\left\{\rho \in \Omega^{2}(M) \mid \rho^{n} \neq 0, d \rho=0,[\rho]=a\right\} .
$$

This is an open set in the Fréchet space of all closed 2-forms on $M$ representing the class $a$. The following three questions correspond to the equivalence relations (a), (A), (B). In all three questions assume that $\mathcal{S}_{a}$ is nonempty (respectively that $M$ admits a symplectic form).

Question 1. Is $\mathcal{S}_{a}$ connected?

Question 2. Are any two symplectic forms in $\mathcal{S}_{a}$ diffeomorphic?

Question 3. Are any two symplectic forms on $M$ deformation equivalent?

Section 3 discusses what is known about these questions in some examples. Section 4 includes a brief discussion of the Donaldson geometric flow approach to Question 1 in dimension four (see [12]).

2.3 (Almost complex structures). Denote the set of almost complex structures on $M$ that are compatible with a nondegenerate 2 -form $\rho$ by

$$
\mathcal{J}(M, \rho):=\left\{J: T M \rightarrow T M \mid J^{2}=-\mathbb{1}, \rho(\cdot, J \cdot) \text { is a Riemannian metric }\right\} .
$$

This space is nonempty and contractible. In fact the space of nondegenerate 2 -forms on $M$ is homotopy equivalent to the space of almost complex structures on $M$. (The homotopy equivalence is a smooth map $\rho \mapsto J_{\rho}$, depending on the choice of a background metric, which assigns to every nondegenerate 2-form $\rho$ an almost complex structure $J_{\rho} \in \mathcal{J}(M, \rho)$; see [19, 38].) Thus every nondegenerate 2 -form $\rho$ on $M$ determines a cohomology class

$$
c_{1}(\rho):=c_{1}(T M, J) \in H^{2}(M ; \mathbb{Z}), \quad J \in \mathcal{J}(M, \rho),
$$

called the first Chern class of $\rho$. Consider the set

$$
\mathcal{C}_{a}:=\left\{c_{1}(\rho) \mid \rho \in \mathcal{S}_{a}\right\} \subset H^{2}(M ; \mathbb{Z}) .
$$


If this set contains more than one element, the answer to Question 1 is negative. Moreover, define

$$
\begin{aligned}
\mathcal{C}_{\text {symp }} & :=\left\{c_{1}(\rho) \mid \rho \text { is a symplectic form on } M\right\}=\bigcup_{a} \mathcal{C}_{a}, \\
\mathcal{C} & :=\left\{c_{1}(\rho) \mid \rho \text { is a nondegenerate 2-form on } M\right\} .
\end{aligned}
$$

Further questions: What is the set $\mathcal{C}_{a}$ ? Is $\mathcal{C}_{\text {symp }}=\mathcal{C}$ ? Does the diffeomorphism group act transitively on $\mathcal{C}_{\text {symp }}$ ?

2.4 (Homotopy classes of almost complex structures). Let $M$ be a compact connected oriented smooth four-manifold without boundary. Denote by $\chi$ the Euler characteristic and by $\sigma$ the signature of $M$. By a theorem of $\mathrm{Wu}$ an integral cohomology class $c \in H^{2}(M ; \mathbb{Z})$ is the first Chern class of an almost complex structure on $M$, compatible with the orientation, if and only if it is an integral lift of the second Stiefel-Whitney class and

$$
c^{2}=2 \chi+3 \sigma .
$$

(The necessity of equation (1) is the Hirzebruch signature theorem.) Given such a class $c$, denote by

$$
\mathcal{J}(M, c):=\left\{\begin{array}{l|l}
J \in \operatorname{Aut}(T M) \mid \begin{array}{l}
J^{2}=-1, c_{1}(T M, J)=c, \\
J \text { induces the given orientation }
\end{array}
\end{array}\right\}
$$

the space of almost complex structures on $M$ with first Chern class $c$ and compatible with the orientation of $M$. If $M$ is simply connected then $\mathcal{J}(M, c)$ has precisely two connected components for every $c \in \mathcal{C}$. In general, there is a bijection

$$
\pi_{0}(\mathcal{J}(M, c)) \cong \operatorname{Tor}_{2}\left(H^{2}(M ; \mathbb{Z})\right) \times\left(\mathbb{Z} / 2 \mathbb{Z} \oplus \frac{H^{3}(M ; \mathbb{Z})}{H^{1}(M ; \mathbb{Z}) \cup c}\right)
$$

for every $c \in \mathcal{C}$. (This was pointed out to me a long time ago by Tom Mrowka, but I don't know a reference.) The first factor is the set

$$
\operatorname{Tor}_{2}\left(H^{2}(M ; \mathbb{Z})\right):=\left\{a \in H^{2}(M ; \mathbb{Z}) \mid 2 a=0\right\} .
$$

It characterizes the isomorphism classes of $\operatorname{spin}^{c}$ structures on $T M$ with first Chern class $c$ (see 2.7 and Lawson-Michelsohn [28, Appendix D]). The second factor characterizes the set of homotopy classes of almost complex structures on $M$ whose canonical $\operatorname{spin}^{c}$ structures are isomorphic to a given $\operatorname{spin}^{c}$ structure with first Chern class $c$. (This can be proved with a standard Pontryagin manifold type construction as in Milnor [41, §7].) 
2.5 (The existence problem). A natural question is under which conditions a given cohomology class $a \in H^{2}(M ; \mathbb{R})$ can be represented by a symplectic form. This is the fundamental existence problem in symplectic topology. The obvious necessary conditions are the existence of an almost complex structure on $M$ and, in the case of a closed manifold, the condition $a^{n} \neq 0$. In the open case a theorem of Gromov [17, 19] asserts that the existence of an almost complex structure is also sufficient. In the closed case there are counterexamples in dimension four, based on Seiberg-Witten theory (see Taubes [50]). The simplest example is $M=\mathbb{C P}^{2} \# \mathbb{C P} \mathbb{P}^{2} \# \mathrm{CP}^{2}$ which does not admit a symplectic form because its Seiberg-Witten invariants vanish. In higher dimensions the existence problem is completely open.

2.6 (Diffeomorphism groups). It is also interesting to investigate the topology of the space $\mathcal{S}_{a}$ of symplectic forms in a given cohomology class beyond the question of connectedness. This is closely related to the topology of certain diffeomorphism groups. Let $(M, \omega)$ be a compact symplectic manifold without boundary and denote $a:=[\omega] \in H^{2}(M ; \mathbb{R})$. Consider the diffeomorphism groups

$$
\begin{aligned}
\operatorname{Diff}_{0}(M) & :=\{\varphi \in \operatorname{Diff}(M) \mid \varphi \text { is isotopic to the identity }\}, \\
\operatorname{Diff}_{0}(M, \omega) & :=\left\{\varphi \in \operatorname{Diff}_{0}(M) \mid \varphi^{*} \omega=\omega\right\} .
\end{aligned}
$$

Thus $\operatorname{Diff}_{0}(M, \omega)$ denotes the group of symplectomorphisms of $M$ that are smoothly isotopic to the identity. Care must be taken to distinguish it from the subgroup of all symplectomorphisms of $M$ that are symplectically isotopic to the identity (i.e. the identity component of $\operatorname{Diff}(M, \omega)$ ). Denote by

$$
\mathcal{S}_{\omega}:=\left\{\rho \in \mathcal{S}_{a} \mid \rho \stackrel{(\mathrm{a})}{\sim} \omega\right\}
$$

the connected component of $\omega$ in $\mathcal{S}_{a}$. (Here $\stackrel{(\text { a) }}{\sim}$ denotes the equivalence relation (a) in 2.1.) By Moser isotopy the group $\operatorname{Diff}_{0}(M)$ acts transitively on $\mathcal{S}_{\omega}$. Hence the map $\operatorname{Diff}_{0}(M) \rightarrow \mathcal{S}_{\omega}: \varphi \mapsto \varphi^{*} \omega$ induces a homeomorphism

$$
\operatorname{Diff}_{0}(M) / \operatorname{Diff}_{0}(M, \omega) \cong \mathcal{S}_{\omega} .
$$

Thus the topology of $\mathcal{S}_{\omega}$ is closely related to the topology of the symplectomorphism group. For example, if there exists a symplectomorphism of $(M, \omega)$ that is smoothly, but not symplectically, isotopic to the identity then $\operatorname{Diff}_{0}(M, \omega)$ is not connected and hence $\mathcal{S}_{\omega}$ has a nontrivial fundamental group (see Example 3.12). 
2.7 (Seiberg-Witten theory). Many results about symplectic structures in dimension four rely on Taubes-Seiberg-Witten theory. Here is some relevant background. Let $M$ be a closed oriented smooth four-manifold.

1. Denote the set of equivalence classes of $\operatorname{spin}^{c}$ structures on $M$ by $\operatorname{Spin}^{c}(M)$. The tensor product with complex line bundles defines a free and transitive action $H^{2}(M ; \mathbb{Z}) \times \operatorname{Spin}^{c}(M) \rightarrow \operatorname{Spin}^{c}(M):(e, \Gamma) \mapsto \Gamma_{e} . \operatorname{Each} \Gamma \in \operatorname{Spin}^{c}(M)$ has a first Chern class $c_{1}(\Gamma) \in H^{2}(M ; \mathbb{Z})$ and $c_{1}\left(\Gamma_{e}\right)=c_{1}(\Gamma)+2 e$.

2. Every nondegenerate 2-form $\rho \in \Omega^{2}(M)$, compatible with the orientation, determines a canonical equivalence class of $\operatorname{spin}^{c}$ structures $\Gamma_{\rho} \in \operatorname{Spin}^{c}(M)$, associated to the $\rho$-compatible almost complex structures. It depends only on the homotopy class of $\rho$ and has the first Chern class $c_{1}\left(\Gamma_{\rho}\right)=c_{1}(\rho)$.

3. Every nondegenerate 2-form $\rho \in \Omega^{2}(M)$, compatible with the orientation, determines a homological orientation of $M$, i.e. an orientation $\mathfrak{o}_{\rho}$ of the real vector space $H^{0}(M ; \mathbb{R}) \oplus H^{1}(M ; \mathbb{R}) \oplus H^{2,+}(M ; \mathbb{R})$ (see Donaldson [10]).

4. If $b^{+}=\operatorname{dim} H^{2,+}(M ; \mathbb{R})=1$ then the positive cone

$$
\mathcal{K}:=\left\{a \in H^{2}(M ; \mathbb{R}) \mid a^{2}>0\right\}
$$

has two connected components. If $\rho$ is a symplectic form, compatible with the orientation, let $\kappa_{\rho} \subset \mathcal{K}$ be the connected component containing $[\rho]$.

5. The Seiberg-Witten invariants of $M$ take the form of a map

$$
\operatorname{Spin}^{c}(M) \rightarrow \mathbb{Z}: \Gamma \mapsto \operatorname{SW}(M, \mathfrak{o}, \Gamma) .
$$

This map depends on the choice of a homological orientation $\mathfrak{o}$ of $M$ (see 3 ). Changing the homological orientation reverses the sign of the invariant. When $b^{+}=1$ the Seiberg-Witten invariant also depends on the choice of a connected component $\kappa$ of $\mathcal{K}$ (see 4 ). Changing the connected component is governed by the wall crossing formula of $\mathrm{Li}-\mathrm{Liu}$ [29].

6. Let $\rho$ be a symplectic form, compatible with the orientation, and $a:=[\rho]$. When $b^{+} \geq 2$, Taubes proved in [49, 50] that

$$
\begin{array}{r}
\operatorname{SW}\left(M, \mathfrak{o}_{\rho}, \Gamma_{\rho}\right)=1, \\
\operatorname{SW}\left(M, \mathfrak{o}_{\rho}, \Gamma_{\rho, e}\right) \neq 0 \quad \Longrightarrow \quad a \cdot e \geq 0, \\
\operatorname{SW}\left(M, \mathfrak{o}_{\rho}, \Gamma_{\rho, e}\right) \neq 0 \quad \text { and } \quad a \cdot e=0 \quad \Longrightarrow \quad e=0
\end{array}
$$

for all $e \in H^{2}(M ; \mathbb{Z})$. In [51] Taubes proved that every cohomology class $e$ with $\operatorname{SW}\left(M, \mathfrak{o}_{\rho}, \Gamma_{\rho, e}\right) \neq 0$ is Poincaré dual to a $\rho$-symplectic submanifold of $M$. These results continue to hold when $b^{+}=1$, with $\operatorname{SW}\left(M, \mathfrak{o}_{\rho}, \Gamma_{\rho, e}\right)$ replaced by $\operatorname{SW}\left(M, \mathfrak{o}_{\rho}, \kappa_{\rho}, \Gamma_{\rho, e}\right)$ (see [49, 50, 51, 52, 53]). 
An immediate corollary of Taubes' theorems is the uniqueness of the first Chern class for cohomologous symplectic forms in dimension four.

Corollary A. Let $M$ be a closed smooth four-manifold. Two cohomologous symplectic forms on $M$ have equivalent spinc structures and hence have the same first Chern class.

Proof. Let $\rho, \rho^{\prime}$ be cohomologous symplectic forms on $M$ and $a:=[\rho]=\left[\rho^{\prime}\right]$. Choose the orientation such that $a^{2}>0$ and choose $e$ such that $\Gamma_{\rho^{\prime}}=\Gamma_{\rho, e}$. Assume first that $b^{+} \geq 2$. By (3), $\operatorname{SW}\left(M, \mathfrak{o}_{\rho^{\prime}}, \Gamma_{\rho, e}\right)=\operatorname{SW}\left(M, \mathfrak{o}_{\rho^{\prime}}, \Gamma_{\rho^{\prime}}\right)=1$ and so $\operatorname{SW}\left(M, \mathfrak{o}_{\rho}, \Gamma_{\rho, e}\right) \neq 0$. Hence $a \cdot e \geq 0$ by (4). Interchanging the roles of $\rho$ and $\rho^{\prime}$ gives $a \cdot e \leq 0$, hence $a \cdot e=0$, and hence $e=0$ by (5). This shows that $\Gamma_{\rho^{\prime}}=\Gamma_{\rho, e}=\Gamma_{\rho}$ and $c_{1}\left(\rho^{\prime}\right)=c_{1}(\rho)+2 e=c_{1}(\rho)$. The proof for $b^{+}=1$ is verbatim the same. Include $\kappa_{\rho}$, respectively $\kappa_{\rho^{\prime}}$, as an argument of SW and use the fact that $\kappa_{\rho}=\kappa_{\rho^{\prime}}$, because $[\rho]=\left[\rho^{\prime}\right]$.

The next corollary is a special case of a result by Conolly-Lé-Ono [8]. It strengthens Corollary A in the simply connected case.

Corollary B. Let $M$ be a simply connected closed smooth four-manifold.

(i) Two symplectic forms on $M$ with the same first Chern class and inducing the same orientation on $M$ are homotopic as nondegenerate 2-forms.

(ii) Two cohomologous symplectic forms on $M$ are homotopic as nondegenerate 2-forms.

Proof. (Following Conolly-Lé-Ono [8].) Assume $b^{+}=1$. (The case $b^{+} \geq 2$ is easier.) Fix an orientation of $M$ and an integral lift $c \in H^{2}(M ; \mathbb{Z})$ of the second Stiefel-Whitney class with $c^{2}=2 \chi+3 \sigma$. Let $\mathcal{R}^{c}$ be the set of nondegenerate 2 -forms on $M$ with first Chern class $c$ and compatible with the orientation. Then $\mathcal{R}^{c}$ has precisely two connected components, by (21). Let $\rho, \rho^{\prime} \in \mathcal{R}^{c}$ be symplectic forms. Then $c_{1}\left(\rho^{\prime}\right)=c_{1}(\rho)=c$ and so $\Gamma_{\rho^{\prime}}=\Gamma_{\rho}$, since $M$ is simply connected. By (즌), $\mathrm{SW}\left(M, \mathfrak{o}_{\rho}, \kappa_{\rho}, \Gamma_{\rho}\right)=1=\mathrm{SW}\left(M, \mathfrak{o}_{\rho^{\prime}}, \kappa_{\rho^{\prime}}, \Gamma_{\rho^{\prime}}\right)$. The wall crossing formula in Li-Liu [29] asserts, in the simply connected case, that $\operatorname{SW}(M, \mathfrak{o}, \kappa, \Gamma)-\operatorname{SW}(M, \mathfrak{o},-\kappa, \Gamma)= \pm 1$ whenever $c_{1}(\Gamma)^{2} \geq 2 \chi+3 \sigma$. Since $\operatorname{SW}\left(M, \mathfrak{o}_{\rho}, \kappa_{\rho}, \Gamma_{\rho}\right)-\mathrm{SW}\left(M, \mathfrak{o}_{\rho}, \kappa_{\rho^{\prime}}, \Gamma_{\rho}\right)$ is even, it follows that $\kappa_{\rho^{\prime}}=\kappa_{\rho}$. Hence $\operatorname{SW}\left(M, \mathfrak{o}_{\rho^{\prime}}, \kappa_{\rho}, \Gamma_{\rho}\right)=1=\operatorname{SW}\left(M, \mathfrak{o}_{\rho}, \kappa_{\rho}, \Gamma_{\rho}\right)$, and hence $\mathfrak{o}_{\rho^{\prime}}=\mathfrak{o}_{\rho}$. In [10, Prop. 3.25] and [11, Lemma 6.4] Donaldson proved that there is a free involution on $\pi_{0}\left(\mathcal{R}^{c}\right)$, which reverses the homological orientation. Hence any two symplectic forms in $\mathcal{R}^{c}$ belong to the same connected component. This proves (i). Assertion (ii) follows from (i) and Corollary A. 


\section{Examples}

Example 3.1 (Open manifolds). Let $M$ be a connected noncompact smooth manifold that admits an almost complex structure. Then the hprinciple rules. Namely, a theorem of Gromov asserts that, for every deRham cohomology class $a \in H^{2}(M ; \mathbb{R})$, the inclusion of the space $\mathcal{S}_{a}$ of all symplectic forms representing the class $a$ into the space of all nondegenerate 2 -forms is a homotopy equivalence. (See [17], [19, page 84], [14, Theorem 10.2.2], [38, Theorem 7.34].) This implies the existence statement in 2.5 and shows that the uniqueness problem reduces to topological obstruction theory.

For Euclidean space $M=\mathbb{R}^{2 n}$ the answer to question 3 is positive and the answers to questions 1 and 2 are negative. Namely, there are two homotopy classes of symplectic forms on $\mathbb{R}^{2 n}$, one for each orientation, and in 18 Gromov constructed a symplectic form $\omega$ on $\mathbb{R}^{2 n}$ (for $n \geq 2$ ) such that $\left(\mathbb{R}^{2 n}, \omega\right)$ is not symplectomorphic to any open subset of $\left(\mathbb{R}^{2 n}, \omega_{0}\right)$ with the standard symplectic form (see also [3] and [38, Example 13.8]). By contrast, if $\omega$ is a symplectic form on $\mathbb{R}^{4}$ that agrees with the standard symplectic form $\omega_{0}$ at infinity, then $\left(\mathbb{R}^{4}, \omega\right)$ is symplectomorphic to $\left(\mathbb{R}^{4}, \omega_{0}\right)$ by another theorem of Gromov (see [18] and [39, Theorem 9.4.2]).

Example 3.2 (Closed two-manifolds). Let $M$ be a closed orientable twomanifold. Then the space $\mathcal{S}_{a}$ is nonempty and convex for every nonzero cohomology class $a \in H^{2}(M ; \mathbb{R})$. Since $M$ admits an orientation reversing diffeomorphism, questions 1, 2, and 3 have positive answers.

In higher dimensions the uniqueness problem for symplectic forms on closed manifolds does not reduce to topological obstruction theory. There is often a dramatic difference between the space of nondegenerate two-forms and the space of symplectic forms, as the following examples show.

Example 3.3 (A six-manifold). This example is due to McDuff [32] (see also [39, Theorem 9.7.4]). Here the set $\mathcal{S}_{a}$ is disconnected. However, by an arbitrarily small perturbation of the cohomology class $a$ the two known distinct connected components of $\mathcal{S}_{a}$ merge to a single connected component. McDuff's paper [32] also contains a variant of this construction in dimension eight. Consider the manifold $M:=\mathbb{T}^{2} \times S^{2} \times S^{2}$. Identify the 2-torus with the

product of two circles. For $\theta \in S^{1}$ and $z \in S^{2}$ let $\varphi_{z, \theta}: S^{2} \rightarrow S^{2}$ be the rotation about the axis through $z$ by the angle $\theta$. Consider the diffeomorphism $\psi: M \rightarrow M$ defined by

$$
\psi\left(\theta_{1}, \theta_{2}, z_{1}, z_{2}\right):=\left(\theta_{1}, \theta_{2}, z_{1}, \varphi_{z_{1}, \theta_{1}}\left(z_{2}\right)\right), \quad \theta_{1}, \theta_{2} \in S^{1}, \quad z_{1}, z_{2} \in S^{2} .
$$


It acts as the identity on cohomology. Let $\omega \in \Omega^{2}(M)$ be the product symplectic form, where both $S^{2}$ factors have the same area, and denote

$$
a:=[\omega]=\left[\psi^{*} \omega\right] \in H^{2}(M ; \mathbb{R}) .
$$

McDuff's theorem asserts that $\omega$ and $\psi^{*} \omega$ can be joined by a path of symplectic forms, but not by a path of cohomologous symplectic forms. The proof that $\omega$ and $\psi^{*} \omega$ are not isotopic cannot be based on the Gromov-Witten invariants because these are invariant under deformation of symplectic forms (equivalence relation (B) in 2.1). McDuff's proof does involve the moduli space of holomorphic spheres. The relevant evaluation maps represent the same homology class but are not homotopic; they have different Hopf invariants. The argument breaks down for symplectic forms where the $S^{2}$ factors have different areas, because in that case the relevant moduli spaces are no longer compact.

Conclusion. For the six-manifold $M=\mathbb{T}^{2} \times S^{2} \times S^{2}$ question 1 has a negative answer and questions 2 and 3 are open problems.

Example 3.4 (The projective plane). A theorem of Taubes [51, 52, 53] asserts that any two symplectic forms on $M=\mathbb{C P}^{2}$ with the same volume are diffeomorphic. The proof uses a theorem of Gromov [18] which requires, as an additional hypothesis, the existence of a symplectically embedded two-sphere (see also [39, Theorem 9.4.1]). The existence of the required symplectic sphere follows from Taubes' "Seiberg-Witten equals Gromov" theorem [53]. Combining Taubes' theorem with Moser isotopy one finds that, for every cohomology class $a \in H^{2}(M ; \mathbb{R})$ with $a^{2} \neq 0$, there is a bijection

$$
\mathcal{S}_{a} \cong \operatorname{Diff}(M, a) / \operatorname{Diff}(M, \omega),
$$

where $\omega$ is the Fubini-Study form representing the class $a$ and

$$
\begin{aligned}
\operatorname{Diff}(M, a) & :=\left\{\varphi \in \operatorname{Diff}(M) \mid \varphi^{*} a=a\right\} \\
& =\left\{\varphi \in \operatorname{Diff}(M) \mid \varphi_{*}=\mathrm{id}: H_{*}\left(\mathbb{C P}^{2} ; \mathbb{Z}\right) \rightarrow H_{*}\left(\mathbb{C P}^{2} ; \mathbb{Z}\right)\right\} .
\end{aligned}
$$

Another theorem of Gromov [18] asserts that $\operatorname{Diff}(M, \omega)$ retracts onto the isometry group PU(3) of $\mathbb{C P}^{2}$ (see also [39, Theorem 9.5.3]).

Conclusion. Questions 2 and 3 have positive answers. Moreover, $\mathcal{S}_{a}$ is connected if and only if every diffeomorphism of $\mathbb{C P}^{2}$ that acts as the identity on homology is isotopic to the identity. 
Example 3.5 (The product $S^{2} \times S^{2}$ ). The discussion of Example 3.4 carries over to $M:=S^{2} \times S^{2}$ as follows. First, every class $a \in H^{2}(M ; \mathbb{R})$ with $a^{2} \neq 0$ is represented by a symplectic form. Second, theorems of Gromov [18] and McDuff [33] assert that every symplectic form for which the homology classes $A:=\left[S^{2} \times\{\mathrm{pt}\}\right]$ and $B:=\left[\{\mathrm{pt}\} \times S^{2}\right]$ (with either orientation) are represented by symplectically embedded spheres is diffeomorphic to a standard form (see [39, Theorem 9.4.7]). Third, Taubes' theorem [53] establishes the existence of the required symplectic spheres. Fourth, a theorem of Gromov [18] asserts that the group of symplectomorphisms that preserve $A, B$ retracts onto the isometry group $\mathrm{SO}(3) \times \mathrm{SO}(3)$ when $A, B$ have the same area (see [39, Theorem 9.5.1]). Fifth, a theorem of Abreu and McDuff [1, 2] asserts that the symplectomorphism group is connected when $A, B$ have different areas. Thus $\mathcal{S}_{a} \cong \operatorname{Diff}(M, a) / \operatorname{Diff}(M, \omega)$ and $\operatorname{Diff}(M, \omega)$ has two connected components when $\langle a, A\rangle=\langle a, B\rangle$, and is connected otherwise.

Conclusion. Questions 2 and 3 have positive answers. Moreover, $\mathcal{S}_{a}$ is connected if and only if every diffeomorphism of $S^{2} \times S^{2}$ that acts as the identity on homology is isotopic to the identity.

Example 3.6 (Ruled surfaces). Let $M$ be an orientable smooth fourmanifold that admits the structure of a fibration over a closed orientable surface $\Sigma$ of positive genus with fibers diffeomorphic to the 2 -sphere:

$$
S^{2} \longleftrightarrow M \stackrel{\pi}{\longrightarrow} \Sigma \text {. }
$$

Fix an orientation of $M$ and an orientation of the fibers. Let $F \in H_{2}(M ; \mathbb{Z})$ be the homology class of the fiber. Call a symplectic form $\omega$ on $M$ compatible with the fibration if it restricts to a symplectic form on each fiber. Call it compatible with the orientations if its cohomology class $a$ satisfies

$$
a^{2}>0, \quad\langle a, F\rangle>0 .
$$

Here are some basic facts.

1. By Seiberg-Witten theory [49, 50] a cohomology class $a \in H^{2}(M ; \mathbb{R})$ is represented by a symplectic form if and only if $a^{2} \neq 0$ and $\langle a, F\rangle \neq 0$. Any such cohomology class is uniquely determined by the numbers $a^{2}$ and $\langle a, F\rangle$. 2. A theorem of McDuff [33] (see also [39, Theorem 9.4.1]) shows that every symplectic form on $M$ that admits a symplectically embedded two-sphere in the homology class $F$ or $-F$ is diffeomorphic to one that is compatible with the fibration. The existence of the required symplectic sphere follows from Taubes-Seiberg-Witten theory [53]. 
3. $M$ admits an orientation preserving diffeomorphism that preserves the fibration and reverses the orientation of the fiber, and an orientation reversing diffeomorphisms that preserves the fibration and the orientation of the fiber. Thus every symplectic form on $M$ is diffeomorphic to one that is compatible with the fibration and orientations.

4. A theorem of Lalonde-McDuff [25, 26] asserts that any two symplectic forms $\omega_{0}, \omega_{1}$ on $M$ that are compatible with the fibration and orientations can be joined by a path of symplectic forms. They also proved that the path can be chosen in the same cohomology class when $\left[\omega_{0}\right]=\left[\omega_{1}\right]$. Thus

$$
\omega_{0} \stackrel{(a)}{\sim} \omega_{1} \quad \Longleftrightarrow \quad \omega_{0} \stackrel{(\mathrm{b})}{\sim} \omega_{1} \text { and }\left[\omega_{0}\right]=\left[\omega_{1}\right]
$$

Here (a) and (b) denote the equivalence relations in 2.1.

Conclusion. On a ruled surface any two symplectic forms are deformation equivalent, and diffeomorphic if they represent the same cohomology class. In the latter case they are homotopic if and only if they are isotopic.

Example 3.7 (The one point blow up of the projective plane). Let

$$
M:=\mathbb{C P}^{2} \# \overline{\mathbb{C P}}^{2} \text {. }
$$

Let $L \in H_{2}(M ; \mathbb{Z})$ be the homology class of the line in $\mathbb{C P}^{2}$ and $E \in H_{2}(M ; \mathbb{Z})$ be the class of the exceptional divisor, both with their complex orientations. They have self-intersection numbers $L \cdot L=1$ and $E \cdot E=-1$. Then $M$ admits an orientation reversing diffeomorphism that interchanges $L$ and $E$, an orientation preserving diffeomorphism that preserves $L$ and reverses $E$, and an orientation preserving diffeomorphism that reverses $L$ and preserves $E$. By Taubes' theorem [51, 52, 53] $L$ or $-L$, and $E$ or $-E$, is represented by a symplectically embedded sphere for every symplectic form on $M$. Hence a class $a \in H^{2}(M ; \mathbb{R})$ is represented by a symplectic form if and only if

$$
a^{2} \neq 0, \quad\langle a, L\rangle \neq 0, \quad\langle a, E\rangle \neq 0 .
$$

By the theorems of McDuff [33, 34] and Taubes [53] any two symplectic forms representing the same cohomology class are diffeomorphic, as in Example 3.6. Thus $\mathcal{S}_{a} \cong \operatorname{Diff}(M, a) / \operatorname{Diff}(M, \omega)$. By (17) every diffeomorphism preserving $a$ acts as the identity on homology. Moreover, a theorem of Abreu and McDuff [1, 2] asserts that $\operatorname{Diff}(M, \omega)$ is connected.

Conclusion. Questions 2 and 3 have positive answers. Moreover, $\mathcal{S}_{a}$ is connected if and only if every diffeomorphism of $M$ that acts as the identity on homology is smoothly isotopic to the identity. 
Example 3.8 (The two point blow up of the projective plane). Consider the oriented four-manifold $M:=\mathbb{C P}^{2} \# \overline{\mathbb{C P}}^{2} \# \overline{\mathbb{C P}}^{2}$. Let $L \in H_{2}(M ; \mathbb{Z})$ be the homology class of the line with self-intersection number $L \cdot L=1$ and $E_{1}, E_{2} \in H_{2}(M ; \mathbb{Z})$ be the homology classes of the exceptional divisors with self-intersection numbers $E_{i} \cdot E_{i}=-1$. Then, by (1) with $2 \chi+3 \sigma=7$,

$$
\mathcal{C}=\left\{\begin{array}{l|l}
c=\operatorname{PD}\left(n L-n_{1} E_{1}-n_{2} E_{2}\right) & \begin{array}{l}
n, n_{1}, n_{2} \text { are odd } \\
n^{2}-n_{1}^{2}-n_{2}^{2}=7
\end{array}
\end{array}\right\} .
$$

The set $\mathcal{E}$ of all classes $E \in H_{2}(M ; \mathbb{Z})$ satisfying $E \cdot E=-1$ is given by

$$
\mathcal{E}=\left\{E=m L+m_{1} E_{1}+m_{2} E_{2} \mid m^{2}-m_{1}^{2}-m_{2}^{2}=-1\right\} .
$$

A theorem of Li-Liu [30, 31] asserts the following for every $a \in H^{2}(M ; \mathbb{R})$.

(I) $\mathcal{C}_{\text {symp }}=\mathcal{C}$.

(II) The diffeomorphism group acts transitively on $\mathcal{C}_{\text {symp }}$.

(III) $\mathcal{S}_{a} \neq \emptyset$ if and only if $a^{2}>0$ and $\langle a, E\rangle \neq 0$ for every $E \in \mathcal{E}$.

(IV) $\mathcal{S}_{a} \neq \emptyset \Longrightarrow \# \mathcal{C}_{a}=1$

(V) If $\omega_{0}, \omega_{1}$ are symplectic forms on $M$, then there exists a diffeomorphism $\varphi$ of $M$ such that $\omega_{0}$ and $\varphi^{*} \omega_{1}$ can be joined by a path of symplectic forms.

Assuming Seiberg-Witten theory, assertion (VI) below is Lemma 3.11 in Karshon-Kessler-Pinsonnault [21] and (VII) is Theorem 1.1 in McDuff [35].

(VI) Two symplectic forms $\omega_{0}, \omega_{1}$ on $M$ have the same first Chern class if and only if there exists a diffeomorphism $\varphi$ of $M$, inducing the identity on homology, such that $\omega_{0}$ and $\varphi^{*} \omega_{1}$ can be joined by a path of symplectic forms.

(VII) Two symplectic forms $\omega_{0}, \omega_{1}$ on $M$ represent the same cohomology class if and only if there exists a diffeomorphism $\varphi$ of $M$, inducing the identity on homology, such that $\varphi^{*} \omega_{1}=\omega_{0}$.

In particular, questions 2 and 3 have positive answers. The proofs rely on the following observations.

1. For every $c \in \mathcal{C}$ there exist precisely three classes $E_{1}^{c}, E_{2}^{c}, E_{3}^{c} \in \mathcal{E}$ such that $\left\langle c, E_{i}^{c}\right\rangle=1$. The numbering can be chosen such that

$$
E_{1}^{c} \cdot E_{3}^{c}=E_{2}^{c} \cdot E_{3}^{c}=1, \quad E_{1}^{c} \cdot E_{2}^{c}=0 .
$$

Each class $E_{i}^{c}$ is represented by an embedded sphere. For the standard first Chern class $c_{\text {std }}=\operatorname{PD}\left(3 L-E_{1}-E_{2}\right) \in \mathcal{C}$ these can be chosen as the classes $E_{1}^{c_{\text {std }}}=E_{1}, E_{2}^{c_{\text {std }}}=E_{2}, E_{3}^{c_{\text {std }}}=L-E_{1}-E_{2}$. For general elements $c \in \mathcal{C}$ this follows from the fact that, by the results of Wall [56, 57, 58, 59], the diffeomorphism group acts transitively on $\mathcal{C}$. (See Li-Liu [30, 31] for details.) 
2. By Taubes-Seiberg-Witten theory [51, 52, 53] the homology classes $E_{1}^{c}, E_{2}^{c}, E_{3}^{c}$ are represented by symplectically embedded spheres for every symplectic form on $M$ with first Chern class $c$.

3. A cohomology class $a \in H^{2}(M ; \mathbb{R})$ can be represented by a symplectic form $\omega$ with first Chern class $c_{1}(\omega)=c$ if and only if for every $E \in H_{2}(M ; \mathbb{Z})$

$$
E \cdot E=-1, \quad\langle c, E\rangle=1 \quad \Longrightarrow \quad\langle a, E\rangle>0 .
$$

By 2. condition (9) is necessary for the existence of $\omega$. The converse follows from the Nakai-Moishezon criterion. (See Demazure [9] and also McDuffPolterovich [37] for a direct symplectic proof.)

4. Let $a \in H^{2}(M ; \mathbb{R})$ such that $a^{2}>0$. Close examination shows that there exists a cohomology class $c \in \mathcal{C}$ such that condition (9) holds if and only if $\langle a, E\rangle \neq 0$ for all $E \in \mathcal{E}$. (See Li-Liu [30, 31] for details.)

Assertions (I), (II), (III) follow from 1.-4. and (IV) follows from Corollary A.

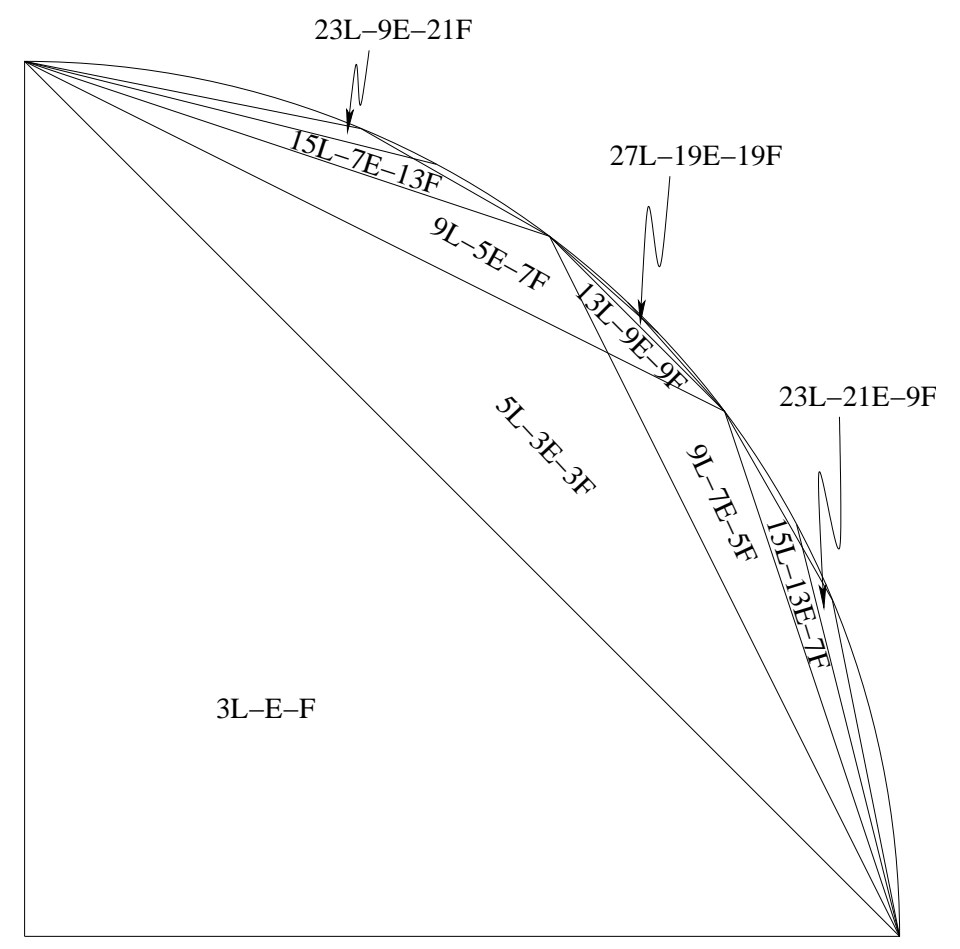

Figure 1: The symplectic chamber structure on $\mathbb{C P}^{2} \# 2 \overline{\mathbb{C P}}^{2}$. 
5. To understand (IV) geometrically, it is convenient to slightly change the point of view. Fix a cohomology class $c \in \mathcal{C}$ and define

$$
\begin{aligned}
& \mathcal{E}^{c}:=\left\{E \in H_{2}(M ; \mathbb{Z}) \mid E \cdot E=-1,\langle c, E\rangle=1\right\} \\
& \mathcal{K}^{c}:=\left\{a \in H^{2}(M ; \mathbb{R}) \mid a^{2}>0,\langle a, E\rangle>0 \forall E \in \mathcal{E}^{c}\right\}, \\
& \mathcal{S}^{c}:=\left\{[\rho] \in H^{2}(M ; \mathbb{R}) \mid \rho \in \Omega^{2}(M), d \rho=0, \rho \wedge \rho>0, c_{1}(\rho)=c\right\} .
\end{aligned}
$$

Then $\mathcal{S}^{c}=\mathcal{K}^{c}$ (see 3 . above). These cones define a chamber structure on $H^{2}(M ; \mathbb{R})$. (See Figure 1 , where the standard basis of $H_{2}(M ; \mathbb{Z})$ is denoted by $L, E, F$ and the symplectic cohomology classes satisfy the normalization conditions $\langle a, L\rangle=1,\langle a, E\rangle>0,\langle a, F\rangle>0$.) The chamber structure is determined by straight lines connecting pairs of rational points on the unit circle. In this notation (IV) asserts that the chambers are disjoint.

6. Here is a sketch of a proof of (V) and (VI), which was worked out in a discussion with Yael Karshon. Construct a model for the symplectic blowup $M=\mathbb{C P} P^{2} \# 2 \overline{\mathbb{C P}}^{2}$ as follows. Choose distinct points $p_{1}, p_{2} \in \mathbb{C P}^{2} \backslash \mathbb{C P}^{1}$ and disjoint neighborhoods $U_{1}, U_{2} \subset \mathbb{C P}^{2} \backslash \mathbb{C P}^{1}$ of $p_{1}, p_{2}$, respectively, equipped with holomorphic coordinate charts

$$
\varphi_{i}: B_{\varepsilon}^{4} \rightarrow U_{i}
$$

defined on the $\varepsilon$ ball in $\mathbb{R}^{4}=\mathbb{C}^{2}$, centered at the origin. Then use the standard complex blowup construction at $p_{1}$ and $p_{2}$ to define $M$ as a complex manifold. There is a holomorphic projection

$$
\pi: M \rightarrow \mathbb{C P}^{2}
$$

with singular values $p_{1}, p_{2}$. Their preimages $S_{1}:=\pi^{-1}\left(p_{1}\right)$ and $S_{2}:=\pi^{-1}\left(p_{2}\right)$ are the exceptional divisors that, with their complex orientations, represent the classes $E_{1}, E_{2}$. The projection $\pi$ is a holomorphic diffeomorphism from $M \backslash\left(S_{1} \cup S_{2}\right)$ to $\mathbb{C P}^{2} \backslash\left\{p_{1}, p_{2}\right\}$ and the holomorphic sphere $S:=\pi^{-1}\left(\mathbb{C P}^{1}\right)$ represents the class $L$.

To define a symplectic form on $M$, let $\omega_{\mathrm{FS}}$ denote the Fubini-Study form on $\mathbb{C P}^{2}$ such that $\mathbb{C P}^{1}$ has area $\pi$. Choose an exact perturbation $\omega_{0}$ of $\omega_{\mathrm{FS}}$ such that $\varphi_{i}^{*} \omega_{0}$ is standard on $B_{\varepsilon}^{4}$ for $i=1,2$. (Warning: $\omega_{0}$ cannot be equal to $\omega_{\mathrm{FS}}$.) Now use the construction in [38, Lemma 7.15] to obtain a symplectic form $\rho_{0}$ on $M$ such that $\rho_{0}=\pi^{*} \omega_{0}$ on $M \backslash \pi^{-1}\left(U_{1} \cup U_{2}\right)$ and the exceptional spheres $S_{1}, S_{2}$ are symplectic submanifolds of small areas. 
Let $\omega$ be any symplectic form on $M$ with first Chern class $c:=c_{1}(\omega)$ and choose $E_{1}^{c}, E_{2}^{c}, E_{3}^{c}$ as in part 1 above such that (8) holds. Denote

$$
L^{c}:=E_{1}^{c}+E_{2}^{c}+E_{3}^{c}, \quad \lambda_{i}:=\left\langle[\omega], E_{i}^{c}\right\rangle, \quad i=1,2,3,
$$

and assume $\left\langle[\omega], L^{c}\right\rangle=\pi$. By Taubes-Seiberg-Witten theory [53], the classes $E_{1}^{c}, E_{2}^{c}, L^{c}$ are represented by pairwise disjoint symplectically embedded spheres $C_{1}, C_{2}, C$. Hence there exist disjoint Weinstein neighborhoods $N_{1}, N_{2} \subset M \backslash C$ of $C_{1}, C_{2}$ in which the symplectic form is standard (see [38, Theorem 3.30]). The symplectic blowdown construction replaces $C_{1}, C_{2}$ by closed four-balls of radii $r_{1}, r_{2}$ with $\pi r_{i}^{2}=\lambda_{i}$. This results in a symplectic manifold $(\widehat{M}, \widehat{\omega})$, equipped with symplectic embeddings $\widehat{\psi}_{i}: B_{r_{i}+\delta}^{4} \rightarrow \widehat{M}$ of closed four-balls with images $\widehat{N}_{i}:=\widehat{\psi}_{i}\left(B_{r_{i}+\delta}^{4}\right)$, and a symplectomorphism

$$
\widehat{\pi}:\left(M \backslash\left(N_{1} \cup N_{2}\right), \omega\right) \rightarrow\left(\widehat{M} \backslash\left(\widehat{N}_{1} \cup \widehat{N}_{2}\right), \widehat{\omega}\right)
$$

Thus $C$ descends to a symplectically embedded sphere $\widehat{C}:=\widehat{\pi}(C) \subset \widehat{M}$, disjoint from $\widehat{N}_{1} \cup \widehat{N}_{2}$, with area $\pi$ and self-intersection number $\widehat{C} \cdot \widehat{C}=1$. The homology class of $\widehat{C}$ generates $H_{2}(\widehat{M} ; \mathbb{Z})$. Hence a theorem of Gromov [18] and McDuff [33] (see also [39, Theorem 9.4.1]) asserts that there exists a symplectomorphism $g:(\widehat{M}, \widehat{C}, \widehat{\omega}) \rightarrow\left(\mathbb{C P}^{2}, \mathbb{C P}^{1}, \omega_{0}\right)$. Consider the embeddings $\psi_{i}:=g \circ \widehat{\psi}_{i}: B_{r_{i}+\delta}^{4} \rightarrow \mathbb{C P}^{2}$. Composing $g$ with a suitable Hamiltonian isotopy we may assume without loss of generality that $\psi_{i}(0)=p_{i}$ and that $\psi_{i}$ agrees with $\varphi_{i}$ near the origin. Thus $g\left(\widehat{N}_{i}\right)=\psi_{i}\left(B_{r_{i}+\delta}^{4}\right)$ is a neighborhood of $p_{i}$ and the complement of their union is symplectomorphic to a subset of $M$ under $\pi^{-1}$. The formula in the proof of [38, Lemma 7.15] gives rise to a diffeomorphism $f: M \rightarrow M$ such that $f\left(C_{i}\right)=S_{i}=\pi^{-1}\left(p_{i}\right), f\left(N_{i}\right)=\pi^{-1}\left(g\left(\widehat{N}_{i}\right)\right)$ and $\left.f\right|_{M \backslash\left(N_{1} \cup N_{2}\right)}=\pi^{-1} \circ g \circ \widehat{\pi}$. The diffeomorphism $f$ satisfies

$$
f_{*} L^{c}=L, \quad f_{*} E_{1}^{c}=E_{1}, \quad f_{*} E_{2}^{c}=E_{2} .
$$

The symplectic form $\rho:=\left(f^{-1}\right)^{*} \omega$ agrees with $\rho_{0}$ on $M \backslash \pi^{-1}\left(g\left(\widehat{N}_{1}\right) \cup g\left(\widehat{N}_{2}\right)\right)$. It can be joined to $\rho_{0}$ by a path of symplectic forms via a scaling argument. If $c_{1}(\omega)=c_{\text {std }}$ one can choose $E_{i}^{c}=E_{i}$ and then $f$ induces the identity on homology. This proves (V) and (VI).

7. The proof of (VII) hinges on the fact that (6) holds for the two point blowup of $\mathbb{C P}^{2}$ and symplectic forms with the standard first Chern class (i.e. two cohomologous symplectic forms with first Chern class $c_{\text {std }}$ are homotopic if and only if they are isotopic). This was proved by McDuff in [34, 35]. Thus (VII) follows from (VI) and Corollary A. 
Example 3.9 (Del Pezzo surfaces). The discussion in Example 3.8 carries over almost verbatim to all del Pezzo surfaces

$$
M:=\mathbb{C P}^{2} \# k \overline{\mathbb{C P}}^{2}, \quad 1 \leq k \leq 8 .
$$

1. The characterization of the symplectic cone $\mathcal{S}^{c}=\mathcal{K}^{c}$ in (10) remains unchanged and the number of exceptional classes is

$$
\#\left\{E \in H_{2}(M ; \mathbb{Z}) \mid \begin{array}{l}
E \cdot E=-1, \\
\langle c, E\rangle=1
\end{array}\right\}=\left\{\begin{aligned}
1, & \text { if } k=1, \\
3, & \text { if } k=2, \\
6, & \text { if } k=3, \\
10, & \text { if } k=4, \\
16, & \text { if } k=5, \\
27, & \text { if } k=6, \\
56, & \text { if } k=7, \\
240, & \text { if } k=8 .
\end{aligned}\right.
$$

(For $k=6$ these are the 27 lines on a cubic in $\mathbb{C P}^{3}$.) Thus condition (9) is still necessary and sufficient for the existence of a symplectic form that represents the class $a$ and has first Chern class $c$. That the condition is necessary follows again from Taubes-Seiberg-Witten theory [53]. That the condition is also sufficient for the standard class

$$
c_{\mathrm{std}}:=\operatorname{PD}\left(3 L-E_{1}-\cdots-E_{k}\right)
$$

is a classical result in Kähler geometry. That the condition is sufficient in general, was proved by Li-Liu [30, 31, by reducing the assertion to the standard chamber via the results of Wall [56, 57, 58].

2. Assertion (6) continues to hold for all blowups of $\mathbb{C P}^{2}$, i.e. two cohomologous symplectic forms are homotopic if and only if they are isotopic. This was first proved by McDuff [34, 35] for $k \leq 2$, then by Biran [4] for $3 \leq k \leq 6$, then by McDuff [36] for all $k$. The proofs (for $k \geq 3$ ) are based on the inflation techniques of Lalonde-McDuff [24, 25, 26, 36].

3. With this understood it follows by the same arguments as in Example 3.8 , parts 6 and 7, that assertions (V), (VI), (VII) continue to hold for all del Pezzo surfaces. (See Li-Liu [30, 31] and Karshon-Kessler-Pinsonnault [21].) Conclusion. Assertions (I)-(VII) in 3.8 remain valid for all del Pezzo surfaces. Thus questions 2 and 3 have positive answers and $\mathcal{C}_{\text {symp }}=\mathcal{C}$. Two cohomologous symplectic forms are homotopic if and only if they are isotopic. 
Example 3.10 (Higher blowups of the projective plane). Consider the closed oriented smooth four-manifolds $M:=\mathbb{C P}^{2} \# k \overline{\mathbb{C P}}^{2}, k \geq 9$.

1. When $k \geq 9$ it is still true that a class $a \in H^{2}(M ; \mathbb{R})$ is represented by a symplectic form with first Chern class $c=c_{\text {std }}$ if and only if $a^{2}>0$ and (9) holds. Thus, in the notation of (10), the symplectic cone is given by $\mathcal{S}^{c_{\text {std }}}=\mathcal{K}^{c_{\text {std }}}$. For $k>9$ this no longer follows from Kähler geometry. It is an existence theorem in symplectic topology by $\mathrm{Li}-\mathrm{Liu}$ [31. An earlier theorem by Biran [5] asserts that $\mathcal{K}^{c_{\text {std }}} \subset \mathcal{S}^{c_{\text {std }}} \subset \overline{\mathcal{K}}^{c_{\text {std }}}$. The proofs are based on Taubes-Seiberg-Witten theory and the inflation techniques developed by Lalonde and McDuff [24, 25, 26, 35, 36]. The Nagata conjecture asserts that the Kähler cone for the standard complex structure should agree with the symplectic cone. (See Biran [7] for a symplectic approach to this question.)

2. Consider the elliptic surface $M:=E(1):=\mathbb{C P}^{2} \# 9 \overline{\mathbb{C P}}^{2}$. This manifold admits the structure of a holomorphic Lefschetz fibration over $\mathbb{C P}^{1}$ with elliptic curves as regular fibers and twelve singular fibers. (Choose two cubics in general position and blow up their nine points of intersection.) In the standard basis $L, E_{1}, \ldots, E_{9}$ of $H_{2}(M ; \mathbb{Z})$ the homology class of the fiber is $F:=3 L-E_{1}-E_{2}-\cdots-E_{9}$. Its Poincaré dual $c_{\text {std }}=\operatorname{PD}(F)=c_{1}\left(T M, J_{\text {std }}\right)$ is the first Chern class of the standard complex structure.

3. For $k \geq 9$ the diffeomorphism group no longer acts transitively on $\mathcal{C}$, however, it acts transitively on $\mathcal{C}_{\text {symp }} \subsetneq \mathcal{C}$. For $k=9$ the theory of Wall [58] is still applicable and shows that, for every $c \in \mathcal{C}$, there is a unique odd integer $m \geq 1$ such that $c$ is diffeomorphic to $m c_{\text {std }}$. By Taubes' results, $c$ is the first Chern class of a symplectic form if and only if $m=1$. For $k \geq 9$, Li-Liu proved in [31, Theorem 1] that

$$
\mathcal{C}_{\text {symp }}=\left\{c \in \mathcal{C} \mid \exists \varphi \in \operatorname{Diff}(M) \text { s.t. } \varphi^{*} c=c_{\text {std }}\right\} .
$$

In [30, Theorem D] they showed that any two symplectic forms with the standard first Chern class are deformation equivalent. Assuming (11), the proof of (V)-(VII) for all $k$ follows the same line of argument as in Example 3.8, parts 6 and 7 , in the case $k=2$. (See Karshon-Kessler-Pinsonnault [21].)

4. There is a chamber structure on $H^{2}(M ; \mathbb{R})$ as in Examples 3.8 and 3.9 . However, the set $\mathcal{E}^{c}$ is now infinite for each $c \in \mathcal{C}_{\text {symp }}$. For more details see Biran [5, 6, 7] and Li-Liu [30, 31].

Conclusion. Assertions (II)-(VII) in 3.8 remain valid for $k \geq 9$. Thus questions 2 and 3 have positive answers. However, $\mathcal{C}_{\text {symp }} \subsetneq \mathcal{C}$. Two cohomologous symplectic forms are homotopic if and only if they are isotopic. 


\section{Example 3.11 (Deformation equivalence).}

1. This example is due to Smith [47]. Consider the four-manifolds

$$
X:=E(5):=E(1) \#_{\mathbb{T}^{2}} E(1) \#_{\mathbb{T}^{2}} E(1) \#_{\mathbb{T}^{2}} E(1) \#_{\mathbb{T}^{2}} E(1), \quad Y:=Z_{5} \# 5 \overline{\mathbb{C P}}^{2},
$$

where $Z_{5} \subset \mathbb{C P}^{3}$ is a degree five hypersurface. Then $X$ admits the structure of a Lefschetz fibration with generic fiber $F$ a torus and 60 singular fibers. Both manifolds are simply connected, not spin, and have the Betti numbers

$$
b^{+}=9, \quad b^{-}=49, \quad \sigma=-40, \quad \chi=60 .
$$

Hence they are homeomorphic and hence $X \times X$ and $Y \times Y$ are diffeomorphic. However, the first Chern class of $X$ with its standard Kähler structure is divisible by three (it is $-3 \mathrm{PD}(F)$ ), while the first Chern class of $Y$ is primitive. These divisibility properties carry over to the product. Hence there exist symplectic forms $\omega_{X} \in \Omega^{2}(X \times X)$ and $\omega_{Y} \in \Omega^{2}(Y \times Y)$ on diffeomorphic manifolds such that

$$
c_{1}\left(\omega_{X}\right) \neq \varphi^{*} c_{1}\left(\omega_{Y}\right)
$$

for every diffeomorphism $\varphi: X \times X \rightarrow Y \times Y$. This gives rise to two symplectic forms on the same manifold that are not even related by the weakest equivalence relation (D) in 2.1. Hence they are not deformation equivalent.

2. In [47, 48] Smith constructed a simply connected four-manifold $X$ that admits two symplectic forms $\omega_{0}, \omega_{1}$ such that $c_{1}\left(\omega_{0}\right)$ is divisible by three and $c_{1}\left(\omega_{1}\right)$ is primitive. His four-manifold is obtained by forming a fiber connected sum of $\mathbb{T}^{4}$ with five copies of $E(1)$. As in 1 . above the first Chern classes of $\omega_{0}$ and $\omega_{1}$ are not diffeomorphic, and hence $\omega_{0}$ and $\omega_{1}$ are not deformation equivalent. In fact, for each integer $N$ he constructed a simply connected four-manifold $X_{N}$ with at least $N$ different deformation equivalence classes of symplectic forms, distinguished by the divisibility properties of their first Chern classes. Taking the product $X_{N} \times \mathbb{T}^{2 n}$, one obtains a $(4+2 n)$-manifold with $N$ pairwise deformation inequivalent symplectic forms.

3. An earlier example of a symplectic four-manifold with two deformation inequivalent symplectic forms was constructed by McMullen-Taubes [40]. In their example the symplectic forms are distinguished by their Seiberg-Witten invariants (see also [55]). In [54] Vidussi constructed homotopy K3's with arbitrarily many deformation inequivalent symplectic forms.

4. In all these examples it is not clear whether two deformation inequivalent symplectic forms can be found in the same cohomology class. 
Example 3.12 (Dehn-Seidel twists). In his PhD thesis Seidel constructed symplectomorphisms on many symplectic four-manifolds $(M, \omega)$ that are smoothly, but not symplectically, isotopic to the identity. He proved the following two theorems (see [44] and [46, Cor $2.9 \&$ Thm 0.5]).

Theorem A. Let $M$ be a closed symplectic four-manifold with Betti numbers

$$
b_{1}:=\operatorname{dim} H^{1}(M ; \mathbb{R})=0, \quad b_{2}:=\operatorname{dim} H^{2}(M ; \mathbb{R}) \geq 3 .
$$

Assume $M$ is minimal (i.e. it does not contain a symplectically embedded two-sphere with self-intersection number minus one).

If $\Lambda \subset M$ is a Lagrangian sphere, then the square

$$
\varphi=\tau_{\Lambda} \circ \tau_{\Lambda}
$$

of the Dehn-Seidel twist determined by $\Lambda$ is smoothly, but not symplectically, isotopic to the identity.

Theorem B. Let $M$ be an algebraic surface and a complete intersection, not diffeomorphic to $\mathbb{C P}^{2}$ or $\mathbb{C P}^{1} \times \mathbb{C P}^{1}$. Then there is a symplectomorphism $\varphi: M \rightarrow M$ that is smoothly, but not symplectically, isotopic to the identity.

Here are some remarks. For a much more detailed and wide ranging discussion of examples and ramifications of these theorems see Seidel [46].

1. The assumptions of Theorem A allow for examples of symplectic fourmanifolds that do not admit Kähler structures (see for example GompfMrowka [16] and Fintushel-Stern [15]). The assumptions of Theorem B allow for examples that are not minimal, such as the six-fold blowup of the projective plane (cubics in $\mathbb{C P}^{3}$ ).

2. The symplectomorphism $\varphi=\tau_{\Lambda}^{2}$ in Theorem $\mathrm{A}$ is smoothly isotopic to the identity by an isotopy localized near $\Lambda$. Seidel computed the Floer cohomology group $\mathrm{HF}^{*}\left(\tau_{\Lambda}\right)$ with its module structure over the quantum cohomology ring, via his exact sequence [44, 45]. As a result he was able to show that, under the assumptions of minimality and $b_{2} \geq 3$, the Floer cohomology group $\operatorname{HF}^{*}\left(\tau_{\Lambda}\right)$ is not isomorphic to the Floer homology group

$$
\operatorname{HF}_{*}\left(\tau_{\Lambda}\right)=\operatorname{HF}^{*}\left(\tau_{\Lambda}^{-1}\right)
$$

Hence $\tau_{\Lambda}$ and $\tau_{\Lambda}^{-1}$ are not Hamiltonian isotopic. When $b_{1}=0$ it then follows that they are not symplectically isotopic. 
3. The assumption $b_{2} \geq 3$ cannot be removed in Theorem A. An example is $M=\mathbb{C P}^{1} \times \mathbb{C P}^{1}$ with its monotone symplectic structures and with $\Lambda$ equal to the anti-diagonal. In this example $\varphi=\tau_{\Lambda}^{2}$ is symplectically isotopic to the identity, by Gromov's theorem in [18] (see Example 3.5).

4. The assumption of minimality cannot be removed in Theorem A. Examples are the blowups $\mathbb{C P}^{2} \# k \overline{\mathbb{C P}}^{2}$ with $2 \leq k \leq 4$. On these manifolds there exist symplectic forms (monotone in the cases $k=3,4$ ) and Lagrangian spheres such that the squares of the Dehn-Seidel twists are symplectically isotopic to the identity (see [46, Examples 1.10 and 1.12]). In contrast, for $5 \leq k \leq 8$, the square of a Dehn-Seidel twist in the $k$-fold blowup of the projective plane with its monotone symplectic form is never symplectically isotopic to the identity (see [46, Example 2.10]).

5. Seidel showed that, by an arbitrarily small perturbation of the cohomology class of $\omega$, the square of the Dehn-Seidel twist deforms to a symplectomorphism that is symplectically isotopic to the identity.

6. If $(M, \omega)$ is a symplectic four-manifold satisfying the assumptions of Theorem A or Theorem B then the space $\mathcal{S}_{\omega}$ of all symplectic forms $\rho$ on $M$ that are isotopic to $\omega$ is not simply connected (see 2.6). However, the nontrivial loops in $\mathcal{S}_{\omega}$ that arise from Dehn-Seidel twists are contractible in the space of nondegenerate 2 -forms.

7. In [23] Kronheimer used Seiberg-Witten theory to prove the existence of symplectic four-manifolds $(M, \omega)$ such that $\mathcal{S}_{\omega}$ is not simply connected. In fact, he developed a method for constructing, for each integer $n \geq 1$, symplectic four-manifolds $(M, \omega)$ such that $\pi_{2 n-1}\left(\mathcal{S}_{\omega}\right) \neq 0$.

Example 3.13 (The K3-surface). As a smooth manifold the K3-surface can be constructed as the fiber connected sum

$$
K 3:=E(2)=E(1) \#_{\mathbb{T}^{2}} E(1) .
$$

On the K3-surface with its standard orientation, every cohomology class $a \in H^{2}(K 3 ; \mathbb{R})$ with $a^{2}>0$ is represented by a symplectic form (with an associated hyperkähler structure). By Taubes' results [49, 50], every symplectic form on the K3-surface with the standard orientation has first Chern class zero (see 2.7). Hence it follows from Corollary B in Section 2 that any two symplectic forms on the K3-surface, compatible with the standard orientation, are homotopic as nondegenerate 2-forms. 
Example 3.14 (The four-torus). Every cohomology class $a \in H^{2}\left(\mathbb{T}^{4} ; \mathbb{R}\right.$ ) with $a^{2} \neq 0$ is represented by a symplectic form and, by Taubes' result in [49, every symplectic form on $\mathbb{T}^{4}$ has first Chern class zero (see 2.7). There are infinitely many homotopy classes of nondegenerate 2-forms with first Chern class zero and compatible with a fixed orientation. The set of such homotopy classes is in bijective correspondence to the set $\mathbb{Z} / 2 \mathbb{Z} \times H^{3}\left(\mathbb{T}^{4} ; \mathbb{Z}\right)$ (see 2.4). The Conolly-Lé-Ono argument in [8] removes only half of these classes as candidates for containing a symplectic form (see Corollary B in Section 2). It remains an open question whether any two symplectic forms on $\mathbb{T}^{4}$, that induce the same orientation, are homotopic as nondegenerate 2-forms.

Example 3.15 (The one point blowup of the four-torus). A recent theorem by Latschev-McDuff-Schlenk [27] asserts that a closed four-ball admits a symplectic embedding into the four-torus with any constant coefficient symplectic form if and only if the volume of the four-ball is smaller than the volume of the four-torus. This settles the existence problem for the one point blowup of the four-torus

$$
M:=\mathbb{T}^{4} \# \overline{\mathbb{C P}}^{2} .
$$

Namely, let $E \in H_{2}(M ; \mathbb{Z})$ be the homology class of the exceptional divisor. Then a cohomology class $a \in H^{2}(M ; \mathbb{R})$ can be represented by a symplectic form if and only if

$$
a^{2} \neq 0, \quad\langle a, E\rangle \neq 0 .
$$

Thus the symplectic cone of $M$ is strictly bigger than the Kähler cone. The uniqueness problem for symplectic forms on $M$ is still far from understood.

Example 3.16 (Kähler surfaces of general type). Let $(M, \omega, J)$ be a minimal Kähler surface of general type with first Chern class $c:=c_{1}(\omega)$ such that

$$
b^{+} \geq 2, \quad c^{2}>0, \quad c \cdot[\omega]<0 .
$$

Then $\pm c$ are the only Seiberg-Witten basic classes. Hence, by Taubes' results in [49], every symplectic form $\rho$ on $M$ satisfies

$$
c_{1}(\rho) \cdot[\rho]<0, \quad c_{1}(\rho)= \pm c .
$$

Thus any two symplectic forms, compatible with the orientation, have the same first Chern class up to sign. It is an open question whether every cohomology class a with $a^{2}>0$ and $a \cdot c<0$ can be represented by a symplectic form with first Chern class $c$ (as conjectured by Tianjun Li). 


\section{Discussion}

Here are some of the conclusions that can be drawn from the examples in Section 3 about the equivalence relations in 2.1 .

1. Many symplectic manifolds (with nonzero first Chern classes) admit antisymplectic involutions. Thus two symplectic forms related by (A) in 2.1 (a diffeomorphism) need not be related by (d) (same first Chern class).

2. Example 3.3 shows that two cohomologous and diffeomorphic symplectic forms on a closed manifold $M$ that can be joined by a path of symplectic forms need not be isotopic. Thus

$$
\left[\omega_{0}\right]=\left[\omega_{1}\right], \quad \omega_{0} \stackrel{(\mathrm{A})}{\sim} \omega_{1}, \quad \omega_{0} \stackrel{(\mathrm{b})}{\sim} \omega_{1} \quad \not \quad \omega_{0} \stackrel{(\mathrm{a})}{\sim} \omega_{1} .
$$

However, for ruled surfaces and all blowups of the projective plane it is known that $\left[\omega_{0}\right]=\left[\omega_{1}\right]$ and (b) imply (a) (see Examples 3.4]3.10).

3. It is an open question whether two symplectic forms on a closed manifold that can be joined by a path of nondegenerate 2-forms can always be joined by a path of symplectic forms. In other words, it is an open question whether the equivalence relations (b) and (c) agree. For open manifolds they agree.

4. For all closed simply connected smooth four-manifolds with nonzero Euler characteristic, the equivalence relation (c) (homotopic as nondegenerate 2forms) agrees with (d) (same first Chern class), by Corollary B in Section 2 . 5. For all blowups of $\mathbb{C P}^{2}$ the relations (c) and (d) agree with a stronger form of (B) (a diffeomorphism acting as the identity on homology, followed by a path of symplectic forms); see 3.8 3.10. The symplectic forms on these manifolds have only one equivalence class with respect to (B), (C), (D).

6. In dimensions $2 n \geq 6$ it is an open question whether two cohomologous symplectic forms on a closed manifold always have the same first Chern class. For closed oriented smooth four-manifolds it follows from Seiberg-Witten theory that $\left[\omega_{0}\right]=\left[\omega_{1}\right]$ implies $c_{1}\left(\omega_{0}\right)=c_{1}\left(\omega_{1}\right)$ (Corollary A in Section 2).

7. It is an entirely different question whether any two symplectic forms, compatible with the orientation, have diffeomorphic first Chern classes up to sign. Examples with positive answers include all symplectic four-manifolds with $b^{+}=1$ (see Li-Liu [31]) and Examples 3.13.3.16. For negative answers in dimension four and higher see Example 3.11 .

8. Example 3.11, part 2, shows that in any dimension $2 n \geq 4$ there exist closed manifolds with pairs of symplectic forms whose first Chern classes are not diffeomorphic (even up to sign); hence they are not related by (D). 
9. It is an open question whether there is any closed four-manifold $M$ and any cohomology class $a \in H^{2}(M ; \mathbb{R})$ such that $\mathcal{S}_{a}$ is nonempty and connected.

10. It is an open question whether there is any closed four-manifold $M$ and any cohomology class $a \in H^{2}(M ; \mathbb{R})$ such that $\mathcal{S}_{a}$ is disconnected.

\section{Conjectures}

Conjecture 4.1 (Donaldson's four-six conjecture). Let $\sigma$ be a symplectic form on the 2-sphere. Let $\left(X, \omega_{X}\right)$ and $\left(Y, \omega_{Y}\right)$ be closed symplectic four-manifolds. Then $X$ and $Y$ are diffeomorphic if and only if the symplectic six-manifolds $\left(X \times S^{2}, \omega_{X} \times \sigma\right)$ and $\left(Y \times S^{2}, \omega_{Y} \times \sigma\right)$ are deformation equivalent, i.e. there exists a diffeomorphism $\varphi: X \times S^{2} \rightarrow Y \times S^{2}$ such that $\omega_{X} \times \sigma$ and $\varphi^{*}\left(\omega_{Y} \times \sigma\right)$ can be joined by a path of symplectic forms on $X \times S^{2}$.

The examples of Smith [47] in 3.11] show that the 2-sphere in Conjecture 4.1 cannot be replaced by the 2-torus. The conjecture is nontrivial in either direction. When $X$ and $Y$ are not diffeomorphic but $X \times S^{2}$ and $Y \times S^{2}$ are diffeomorphic, the conjecture suggests that the two symplectic structures on these six-manifolds should still remember the differences in the smooth structures on $X$ and $Y$. At the time of writing the only known methods for distinguishing smooth structures on four-manifolds are the Donaldson invariants and the Seiberg-Witten invariants. By Taubes-Seiberg-Witten theory [51, 52, 53] the Seiberg-Witten invariants can also be interpreted as symplectic invariants, and it is conceivable that they give rise to a method for distinguishing the symplectic structures on the products with the twosphere. An example where $X$ and $Y$ are not diffeomorphic, $X \times S^{2}$ and $Y \times S^{2}$ are diffeomorphic, and the symplectic forms on $X \times S^{2}$ and $Y \times S^{2}$ can be distinguished by their Gromov-Witten invariants was found by Ruan [42] (see also [39, Example 9.7.1]). More examples along these lines were found by Ruan-Tian [43] and Ionel-Parker [20].

Conjecture 4.2 (Uniqueness conjecture). Let $M$ be a closed hyperkähler surface (i.e. a four-torus or a $K 3$ surface) and let $a \in H^{2}(M ; \mathbb{R})$ be a cohomology class such that $a^{2}>0$. Then the space $\mathcal{S}_{a}$ of symplectic forms on $M$ representing the class a is connected.

The uniqueness problem for the four-torus is a longstanding open question in symplectic topology, which goes back at least to the early eighties. In [12] Donaldson proposed a remarkable geometric approach to the uniqueness question for symplectic forms on hyperkähler surfaces which I explain next. 


\section{The Donaldson geometric flow}

The starting point of Donaldson's approach is to view the space $\mathscr{F}_{a}$ of diffeomorphisms from a symplectic four-manifold $(S, \sigma)$ to a hyperkähler surface $\left(M, \omega_{1}, \omega_{2}, \omega_{3}, J_{1}, J_{2}, J_{3}\right)$, that pull back the cohomology class $a$ of $\omega_{1}$ to that of $\sigma$, as an infinite dimensional hyperkähler manifold. The group of symplectomorphisms of $(S, \sigma)$ acts on $\mathscr{F}_{a}$ by hyperkähler isometries and the action of the subgroup of Hamiltonian symplectomorphisms is generated by a hyperkähler moment map. The negative gradient flow of the square of the hyperkähler moment map is a parabolic type equation for a path of diffeomorphisms $f_{t} \in \mathscr{F}_{a}$ depending on a real parameter $t$. It has the form

$$
\partial_{t} f_{t}=\sum_{i} J_{i} d f_{t} \circ X_{H_{i}^{f_{t}}}, \quad H_{i}^{f}:=\frac{f^{*} \omega_{i} \wedge \sigma}{\operatorname{dvol}_{\sigma}}, \quad \iota\left(X_{H}\right) \sigma=d H,
$$

where $\operatorname{dvol}_{\sigma}:=\frac{1}{2} \sigma \wedge \sigma$. Equation (12) is preserved by the symplectomorphism group of $(S, \sigma)$. One can elmininate the action of the reparametrization group by pushing forward the sympectic form $\sigma$ under the diffeomorphisms $f_{t}$. The resulting path $\rho_{t}:=\left(f_{t}^{-1}\right)^{*} \sigma \in \mathcal{S}_{a}$ satisfies the equation

$$
\partial_{t} \rho_{t}=-\sum_{i} d\left(d K_{i}^{\rho_{t}} \circ J_{i}^{\rho_{t}}\right), \quad K_{i}^{\rho}:=\frac{\omega_{i} \wedge \rho}{\operatorname{dvol}_{\rho}}, \quad \rho\left(J_{i} \cdot, \cdot\right)=\rho\left(\cdot, J_{i}^{\rho} \cdot\right) .
$$

This is the Donaldson geometric flow. It is the negative gradient flow of the energy functional $\mathcal{E}: \mathcal{S}_{a} \rightarrow \mathbb{R}$ defined by

$$
\mathcal{E}(\rho):=\frac{1}{2} \int_{M} \sum_{i}\left|\frac{\omega_{i} \wedge \rho}{\operatorname{dvol}_{\rho}}\right|^{2} \operatorname{dvol}_{\rho}=2 \int_{M} \frac{\left|\rho^{+}\right|^{2}}{\left|\rho^{+}\right|^{2}-\left|\rho^{-}\right|^{2}} \mathrm{dvol} .
$$

The inner products on the tangent spaces $T_{\rho} \mathcal{S}_{a}=\left\{\widehat{\rho} \in \Omega^{2}(M) \mid \widehat{\rho}\right.$ is exact $\}$ are associated to the norms

$$
\|\widehat{\rho}\|_{\rho}^{2}:=\int_{M}\left|X_{\widehat{\rho}}\right|^{2} \operatorname{dvol}_{\rho}, \quad \widehat{\rho}=-d \iota\left(X_{\widehat{\rho}}\right) \rho, \quad \rho \wedge\left\langle X_{\widehat{\rho}}, \cdot\right\rangle \in \operatorname{im} d .
$$

The gradient flow of the energy functional (14) on $\mathcal{S}_{a}$ with respect to the inner products determined by (15) is meaningful for any closed symplectic four-manifold $(M, \omega)$, equipped with a background Riemannian metric. It is convenient to choose the Riemannian metric on $M$ to be compatible with $\omega$. Then $\omega$ is the unique global minimum of $\mathcal{E}$ on $\mathcal{S}_{a}$ and the Hessian of $\mathcal{E}$ is positive definite at $\omega$. In [12] Donaldson also observed that the energy $\mathcal{E}(\rho)$ controls the $L^{1}$-norm of $\rho$ and he proved, in the hyperkähler case, that the Hessian at any other critical point (if it exists) cannot be positive definite. 
The study of equation (13) poses deep and challenging analytical problems. Already regularity and short time existence are nontrivial. They are the subject of as yet unpublished work by Robin Krom [22], which is based on ideas of Donaldson in [13]. The hope is that, in the hyperkähler setting, one can establish long time existence and convergence for equation (13) and use this to prove that the space $\mathcal{S}_{a}$ of symplectic forms in a fixed cohomology class is connected. This hope is backed up by the fact that an analogous geometric flow approach in dimension two leads to the parabolic equation $\partial_{t} u_{t}=d^{*} d u_{t}^{-1}$ (see [12]). In this equation the proof of long time existence is based on the observation that the time derivative is nonpositive at each local maximum of $u_{t}$ and is nonnegative at each local minimum of $u_{t}$.

Acknowledgement. Thanks to Paul Biran, Simon Donaldson, Yael Karshon, Janko Latschev, Dusa McDuff, and Stefano Vidussi for many helpful comments and suggestions.

\section{References}

[1] M. Abreu, Topology of symplectomorphism groups of $S^{2} \times S^{2}$. Invent. Math. 131 (1998), 1-23.

[2] M. Abreu, D. McDuff, Topology of symplectomorphism groups of rational ruled surfaces. J. Amer. Math. Soc. 13 (2000), 971-1009.

[3] L. Bates, G Peschke, A remarkable symplectic structure. J. Diff. Geom. 32 (1990), 33-38.

[4] P. Biran, Connectedness of spaces of symplectic embeddings. IMRN 1996, 487-491.

[5] P. Biran, Symplectic packing in dimension 4. GAFA 7 (1997), 420-437.

[6] P. Biran, A stability property of symplectic packing. Invent. Math. 136 (1999), 123-155.

[7] P. Biran, From symplectic packing to algebraic geometry and back. European Congress of Mathematics, Barcelone 2000, 507-524. Progress in Math. 202, Birkhäuser, Basel, 2001.

[8] F. Conolly, H.V. Lé, K. Ono, Almost complex structures which are compatible with Kähler or symplectic structures. Ann. Global Anal. Geom. 15 (1997), 325-334.

[9] M.Demazure, Surfaces de del Pezzo II-V. Séminar sur les singularités de surfaces (1976-1977), Lecture Notes in Mathematics 777, Springer, 1980.

[10] S.K. Donaldson, The Orientation of Yang-Mills Moduli Spaces and Four-Manifold Topology. JDG 26 (1987), 397-428.

[11] S.K. Donaldson, Polynomial Invariants of Smooth Four-Manifolds. JDG 29 (1990), 257-315.

[12] S.K. Donaldson, Moment Maps and Diffeomorphisms. Asian J. Math. 3 (1999), 1-16.

[13] S.K. Donaldson, Two-Forms on Four-Manifolds and Elliptic Equations. Inspired by S. S. Chern, edited by Phillip A. Griffiths, Nankai Tracts Mathematics 11, World Scientific, 2006, pages 153-172.

[14] Y. Eliashberg, N. Mishachev, Introduction to the h-Principle. Graduate Studies in Mathematics 48, AMS 2002.

[15] R. Fintushel, R. Stern, Knots, links, and four-manifolds. Invent. Math. 134 (1998), 363-400.

[16] R. Gompf, T. Mrowka, Irreducible four-manifolds need not be complex. Annals of Mathemetics 138 (1993), 61-111.

[17] M. Gromov, Stable Mappings of Foliations into Manifolds. Izv. Akad. Nauk SSSR Ser. Mat. 33 (1969), 707-734.

[18] M. Gromov, Pseudo holomorphic curves in symplectic manifolds. Invent. Math. 82 (1985), 307-347. 
[19] M. Gromov, Partial differential relations. Springer, Berlin, 1986.

[20] E.-N. Ionel, T.H. Parker, Gromov invariants and symplectic maps. Mathematische Annalen 314 (1999), 127-158.

[21] Y. Karshon, L. Kessler, M. Pinsonnault, Symplectic blowups of the complex projective plane and counting toric actions. Preprint, http://www.math.toronto.edu/karshon/, October 2012.

[22] R. Krom, PhD Thesis, ETH Zürich, in preparation.

[23] P.B. Kronheimer, Some nontrivial families of symplectic structures. Preprint, Harvard 1997. http://www.math.harvard.edu/ kronheim/papers.html

[24] F. Lalonde, Isotopy of symplectic balls, Gromov's radius, and the structure of symplectic manifolds. Mathematische Annalen 300 (1994), 273-296.

[25] F. Lalonde, D. McDuff, The classification of ruled symplectic 4-manifolds. Math. Res. Lett. 3 (1996), 769-778.

[26] F. Lalonde, D. McDuff, J-curves and the classification of rational and ruled symplectic 4-manifolds. in: Symplectic and Contact Geometry ed. C. Thomas, Camb. Univ. Press 1996, pp 3-42.

[27] J. Latschev, D. McDuff, F. Schlenk, The Gromov-width of four-dimensional tori. Preprint, November 2011.

[28] H.B. Lawson, M.-L. Michelsohn, Spin Geometry. Princeton Mathematical Series 58, Princeton University Press, 1989.

[29] T.J. Li, A. Liu, General wallcrossing formula, Math. Research Letters 2 (1995), 797-810.

[30] T.J. Li, A. Liu, Symplectic structure on ruled surfaces and generalized adjunction formula. Math. Res. Lett. 2 (1995), 453-471.

[31] T.J. Li, A. Liu, Uniqueness of symplectic canonical class, surface cone and symplectic cone of 4-manifolds with $b^{+}=1$. J. Diff. Geom. 58 (2001), 331-370.

[32] D. McDuff, Examples of symplectic structures. Invent. Math. 89 (1987), 13-36.

[33] D. McDuff, The structure of rational and ruled symplectic 4-manifolds. J. Amer. Math. Soc. 3 (1990), 679-712. Erratum: J. Amer. Math. Soc 5 (1992), 987-988.

[34] D. McDuff, Blowing up and symplectic embeddings. Topology 30 (1991), 409-421.

[35] D. McDuff, Remarks on the uniqueness of symplectic blowing up. Symplectic Geometry, edited by D. Salamon, LMS Lecture Note Series 192, Cambridge University Press 1993, pp 157-168.

[36] D. McDuff, From symplectic deformation to isotopy. Topics in Symplectic Four-Manifolds, edited by R.J. Stern, International Press Lecture Series, Volume 1, Intl. Press, Cambridge, 1998, pp 85-100.

[37] D. McDuff, L. Polterovich, Symplectic packings and algebraic geometry, Invent. Math. 115 (1994), 405-429.

[38] D. McDuff, D. Salamon, Introduction to Symplectic Topology, Second Edition. Oxford University Press, 2005.

[39] D. McDuff, D. Salamon, J-holomorphic Curves and Symplectic Topology, Second Edition. AMS Colloquium Publications 52, 2012.

[40] C.T. McMullen and C.H. Taubes, 4-manifolds with inequivalent symplectic forms and 3-manifolds with inequivalent fibrations. Math. Res. Lett. 6 (1999), 681-696.

[41] J.W. Milnor, Topology from the Differential Viewpoint. The University Press of Virginia, 1965.

[42] Y. Ruan, Symplectic topology on algebraic three-folds. J. Diff. Geom. 39 (1994), 215-227.

[43] Y. Ruan, G. Tian, Higher genus symplectic invariants and sigma models coupled with grqavity. Invent. Math. 130 (1997), 455-516.

[44] P. Seidel, Floer homology and the symplectic isotopy problem. Ph.D. thesis, Oxford University, 1997.

[45] P. Seidel, A long exact sequence for symplectic Floer cohomology. Topology 42 (2003), 1003-1063.

[46] P. Seidel, Lectures on four-dimensional Dehn twists. Symplectic four-manifolds and algebraic surfaces, 231-267, Lecture Notes in Mathematics 1938, Springer, 2008.

[47] I. Smith, On moduli spaces of symplectic forms. Math. Res. Letters 7 (2000), 779-788.

[48] I. Smith, Torus fibrations on symplectic 4-manifolds. Turkish J. Math. 25 (1) (2001), 69-95. 
[49] C.H. Taubes, The Seiberg-Witten invariants and symplectic forms. Math. Res. Lett. 1 (1994), 809822 .

[50] C.H. Taubes, More constraints on symplectic forms from Seiberg-Witten invariants. Math. Res. Lett. 2 (1995), 9-14.

[51] C.H. Taubes, The Seiberg-Witten and the Gromov invariants. Math. Res. Lett. 2 (1995), 221-238.

[52] C.H. Taubes, Counting pseudoholomorphic submanifolds in dimension four. J. Diff. Geom. 44 (1996), 818-893. (republished in 53])

[53] C.H. Taubes, Seiberg-Witten and Gromov Invariants for Symplectic 4-Manifolds. First Internat. Press Lecture Series vol 2, Internat. Press. Cambridge MA, 2000.

[54] Stefano Vidussi, Homotopy K3's with several symplectic structures. Geom. Topol. 5 (2001), 267-285.

[55] Stefano Vidussi, Smooth structure of some symplectic surfaces. Michigan Math. J. 49 (2001), 325330.

[56] C.T.C. Wall, On the orthogonal groups of unimodular quadratic forms. Mathematische Annalen 147 (1962) 328-338.

[57] C.T.C. Wall, On the orthogonal groups of unimodular quadratic forms II. J. Reine und Angew. Math. 213 (1963) 122-136.

[58] C.T.C. Wall, Diffeomorphisms of 4-Manifolds. Journal L.M.S. 39 (1964) 131-140.

[59] C.T.C. Wall, On simply connected 4-Manifolds. Journal L.M.S. 39 (1964) 141-149. 